\title{
Erratum: Higher-order moments, cumulants, and spectra of continuous quantum noise measurements [Phys. Rev. B 98, 205143 (2018)]
}

\author{
Daniel Hägele, Markus Sifft, and Fabian Schefczik
}

(Received 26 August 2020; published 8 September 2020)

DOI: 10.1103/PhysRevB.102.119901

We wrongly claimed in our derivation of the four-time cumulant $C_{4}\left(z\left(t_{1}\right), z\left(t_{2}\right), z\left(t_{3}\right), z\left(t_{4}\right)\right)$ that the terms $\alpha_{13}$ and $\alpha_{31}$ cancel in the original paper. A correct treatment starts with (we use the short notation of Appendix F)

$$
\alpha_{13}+\alpha_{31}=T\left(A G_{43}^{\prime} A \rho_{0}\right) T\left(A G_{21}^{\prime} A \rho_{0}\right) \theta_{32}-T\left(A G_{43}^{\prime} A \rho_{0}\right) T\left(A G_{21}^{\prime} A \rho_{0}\right) / 2 .
$$

The contribution of the sum $\operatorname{Sum}\left(\alpha_{13}+\alpha_{31}\right)$ to $C_{4}$ contains all 24 permutations of the indices 1 to 4 . Assuming a preliminary time-order $t_{4}>t_{3}>t_{2}>t_{1}$, one finds that most terms vanish [e.g., $-T\left(A G_{23}^{\prime} A \rho_{0}\right) T\left(A G_{41}^{\prime} A \rho_{0}\right) / 2$ is zero since $G_{23}^{\prime}=0$ due to $\left.t_{3}>t_{2}\right]$. The surviving terms give rise to

$$
\begin{aligned}
\operatorname{Sum}\left(\alpha_{13}+\alpha_{31}\right) & =-T\left(A G_{42}^{\prime} A \rho_{0}\right) T\left(A G_{31}^{\prime} A \rho_{0}\right)-T\left(A G_{41}^{\prime} A \rho_{0}\right) T\left(A G_{32}^{\prime} A \rho_{0}\right) \\
& =-T\left(A G_{43}^{\prime} G_{32}^{\prime} A \rho_{0}\right) T\left(A G_{32}^{\prime} G_{21}^{\prime} A \rho_{0}\right)-T\left(A G_{43}^{\prime} G_{32}^{\prime} G_{21}^{\prime} A \rho_{0}\right) T\left(A G_{32}^{\prime} A \rho_{0}\right) .
\end{aligned}
$$

The notation in the last line guarantees that, for all time orders other than $t_{4}>t_{3}>t_{2}>t_{1}$, the right-hand side is zero. We, therefore, can relax the restriction $t_{4}>t_{3}>t_{2}>t_{1}$ and are left with

$$
\operatorname{Sum}\left(\alpha_{13}+\alpha_{31}\right)=-\operatorname{Sum}\left[T\left(A G_{43}^{\prime} G_{32}^{\prime} A \rho_{0}\right) T\left(A G_{32}^{\prime} G_{21}^{\prime} A \rho_{0}\right)\right]-\operatorname{Sum}\left[T\left(A G_{43}^{\prime} G_{32}^{\prime} G_{21}^{\prime} A \rho_{0}\right) T\left(A G_{32}^{\prime} A \rho_{0}\right)\right],
$$

which is valid for all time orders. The correct expression for the four-time cumulant [Eq. (100) in our paper] follows as:

$$
\begin{aligned}
C_{4}\left(z\left(t_{1}\right), z\left(t_{2}\right), z\left(t_{3}\right), z\left(t_{4}\right)\right)= & \beta^{8} \sum_{\text {prm. } t_{j}} \operatorname{Tr}\left[\mathcal{A}^{\prime} \mathcal{G}^{\prime}\left(t_{4}-t_{3}\right) \mathcal{A}^{\prime} \mathcal{G}^{\prime}\left(t_{3}-t_{2}\right) \mathcal{A}^{\prime} \mathcal{G}^{\prime}\left(t_{2}-t_{1}\right) \mathcal{A}^{\prime} \rho_{0}\right] \\
& -\beta^{8} \sum_{\operatorname{prm} . t_{j}} \operatorname{Tr}\left[\mathcal{A}^{\prime} \mathcal{G}^{\prime}\left(t_{4}-t_{3}\right) \mathcal{G}^{\prime}\left(t_{3}-t_{2}\right) \mathcal{A}^{\prime} \rho_{0}\right] \operatorname{Tr}\left[\mathcal{A}^{\prime} \mathcal{G}^{\prime}\left(t_{3}-t_{2}\right) \mathcal{G}^{\prime}\left(t_{2}-t_{1}\right) \mathcal{A}^{\prime} \rho_{0}\right] \\
& -\beta^{8} \sum_{\text {prm. } t_{j}} \operatorname{Tr}\left[\mathcal{A}^{\prime} \mathcal{G}^{\prime}\left(t_{4}-t_{3}\right) \mathcal{G}^{\prime}\left(t_{3}-t_{2}\right) \mathcal{G}^{\prime}\left(t_{2}-t_{1}\right) \mathcal{A}^{\prime} \rho_{0}\right] \operatorname{Tr}\left[\mathcal{A}^{\prime} \mathcal{G}^{\prime}\left(t_{3}-t_{2}\right) \mathcal{A}^{\prime} \rho_{0}\right]
\end{aligned}
$$

with additional lines two and three originating from $\alpha_{13}+\alpha_{31} \neq 0$. The superoperator $\mathcal{A}$ in Eq. (3) was replaced in Eq. (4) by $\mathcal{A}^{\prime}=\mathcal{A}-\operatorname{Tr}\left(\mathcal{A} \rho_{0}\right)$ with the same arguments that were given in the original paper before Eq. (99). The corresponding fourthorder spectrum $S^{(4)}$ (trispectrum) [Eq. (109)] follows via Fourier transformation of the four-time $C_{4}$ (compare Sec. XIV) as

$$
\begin{aligned}
& S^{(4)}\left(\omega_{1}, \omega_{2}, \omega_{3}, \omega_{4}=-\omega_{1}-\omega_{2}-\omega_{3}\right) \\
&=\beta^{8} \sum_{\text {prm. } \omega_{1}, \omega_{2}, \omega_{3}, \omega_{4}} \operatorname{Tr}\left[\mathcal{A}^{\prime} \mathcal{G}^{\prime}\left(\omega_{4}\right) \mathcal{A}^{\prime} \mathcal{G}^{\prime}\left(\omega_{3}+\omega_{4}\right) \mathcal{A}^{\prime} \mathcal{G}^{\prime}\left(\omega_{2}+\omega_{3}+\omega_{4}\right) \mathcal{A}^{\prime} \rho_{0}\right] \\
&-\frac{\beta^{8}}{2 \pi} \sum_{\text {prm. } \omega_{1}, \omega_{2}, \omega_{3}, \omega_{4}} \int \operatorname{Tr}\left[\mathcal{A}^{\prime} \mathcal{G}^{\prime}\left(\omega_{4}\right) \mathcal{G}^{\prime}\left(\omega_{3}+\omega_{4}-\omega\right) \mathcal{A}^{\prime} \rho_{0}\right] \operatorname{Tr}\left[\mathcal{A}^{\prime} \mathcal{G}^{\prime}(\omega) \mathcal{G}^{\prime}\left(\omega_{2}+\omega_{3}+\omega_{4}\right) \mathcal{A}^{\prime} \rho_{0}\right] d \omega \\
&-\frac{\beta^{8}}{2 \pi} \sum_{\text {prm. } \omega_{1}, \omega_{2}, \omega_{3}, \omega_{4}} \int \operatorname{Tr}\left[\mathcal{A}^{\prime} \mathcal{G}^{\prime}\left(\omega_{4}\right) \mathcal{G}^{\prime}\left(\omega_{2}+\omega_{3}+\omega_{4}\right) \mathcal{G}^{\prime}\left(\omega_{3}+\omega_{4}-\omega\right) \mathcal{A}^{\prime} \rho_{0}\right] \operatorname{Tr}\left[\mathcal{A}^{\prime} \mathcal{G}^{\prime}(\omega) \mathcal{A}^{\prime} \rho_{0}\right] d \omega,
\end{aligned}
$$

where the additional terms $S^{(4,2)}$ and $S^{(4,3)}$ (lines two and three, respectively) require the calculation of a convolution integral.

The numerical evaluation of, e.g., $S^{(4,3)}$ is, however, more conveniently performed in the following way: $S^{(4,3)}$ is obtained from the function $g_{3}$ (instead of $g$, compare Sec. XIV),

$$
g_{3}\left(\tau_{1}, \tau_{2}, \tau_{3}\right)=-\operatorname{Tr}\left[\mathcal{A}^{\prime} \mathcal{G}^{\prime}\left(\tau_{3}+\tau_{2}+\tau_{1}\right) \mathcal{A}^{\prime} \rho_{0}\right] \operatorname{Tr}\left[\mathcal{A}^{\prime} \mathcal{G}^{\prime}\left(\tau_{2}\right) \mathcal{A}^{\prime} \rho_{0}\right]
$$


(a)

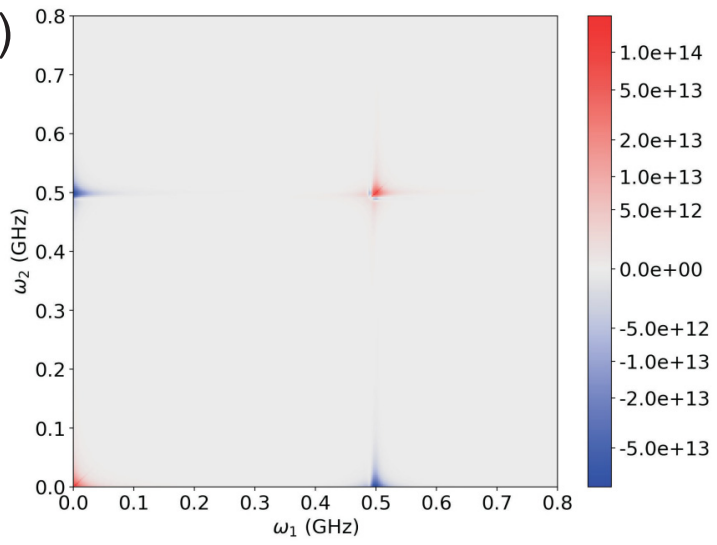

(b)

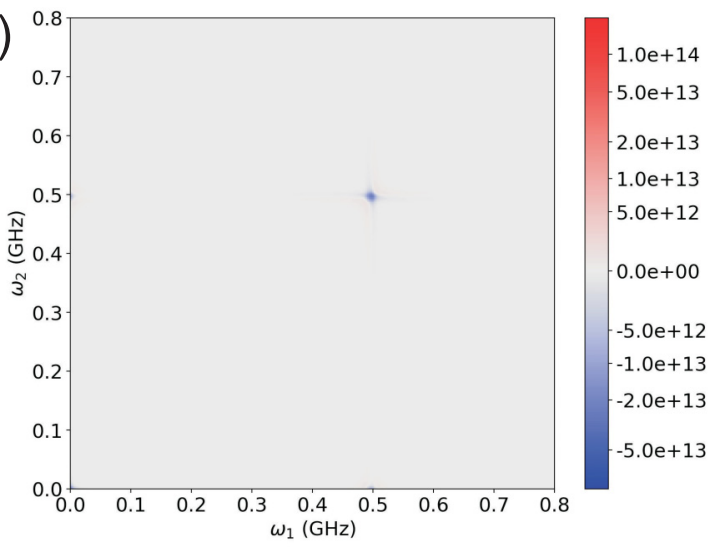

FIG. 1. (a) Replacement of Fig. 4(a): Fourth-order correlation spectrum $S^{(4)}\left(\omega_{1},-\omega_{1}, \omega_{2},-\omega_{2}\right)$ of the spin-spin system in ZnO:In at $B_{x}=0 \mathrm{mT}$. (b) Contribution of $S^{(4,2)}$ and $S^{(4,3)}$.

via its Fourier transformation $\tilde{g}_{3}\left(v_{1}, v_{2}, v_{3}\right)$. For a numerical evaluation of $\tilde{g}_{3}$, we rewrite $g_{3}$ (for a definition of $\Lambda$, see our discussion in Sec. XV),

$$
g_{3}\left(\tau_{1}, \tau_{2}, \tau_{3}\right)=-\beta^{8} \operatorname{Tr}\left[\mathcal{A}^{\prime} \Lambda \mathcal{D}\left(\tau_{3}+\tau_{2}+\tau_{1}\right) \Lambda^{-1} \mathcal{A}^{\prime} \rho_{0}\right] \operatorname{Tr}\left[\mathcal{A}^{\prime} \Lambda \mathcal{D}\left(\tau_{2}\right) \Lambda^{-1} \mathcal{A}^{\prime} \rho_{0}\right]
$$

with diagonal matrix $[\mathcal{D}(\tau)]_{k, k}=e^{\lambda_{k} \tau}$ and $[\mathcal{D}(\tau)]_{m, m}=0$ if $\lambda_{m}=0$. We introduce the matrix $\left(\mathcal{S}_{k}\right)_{i, j}=\delta_{i, k} \delta_{j, k}\left(1-\delta_{m, k}\right)$ with a single nonzero entry at $(k, k)$ for $k \neq m$. After defining

$$
s_{k}=\operatorname{Tr}\left[\mathcal{A}^{\prime} \Lambda \mathcal{S}_{k} \Lambda^{-1} \mathcal{A}^{\prime} \rho_{0}\right]
$$

we can write

$$
g_{3}\left(\tau_{1}, \tau_{2}, \tau_{3}\right)=-\beta^{8} \sum_{k \neq m, l \neq m} s_{k} s_{l} e^{\lambda_{k}\left(\tau_{3}+\tau_{2}+\tau_{1}\right)} e^{\lambda_{l} \tau_{2}} .
$$

The Fourier transformation then obtains the form

$$
\tilde{g}_{3}\left(v_{1}, v_{2}, v_{3}\right)=-\beta^{8} \sum_{k \neq m, l \neq m} s_{k} s_{l} \frac{-1}{\left(\lambda_{k}+i \nu_{1}\right)\left(\lambda_{k}+i \nu_{3}\right)\left(\lambda_{k}+\lambda_{l}+i \nu_{2}\right)} .
$$

Consequently, the contribution $S^{(4,3)}$ to $S^{(4)}$ is (compare Sec. XIV),

$$
S^{(4,3)}\left(\omega_{1}, \omega_{2}, \omega_{3}, \omega_{4}\right)=\left.\beta^{8} \sum_{k \neq m, l \neq m} \sum_{\text {perm. } \omega_{j}} s_{k} s_{l} \frac{1}{\left(\lambda_{k}+i \nu_{1}\right)\left(\lambda_{k}+i \nu_{3}\right)\left(\lambda_{k}+\lambda_{l}+i \nu_{2}\right)}\right|_{\nu_{1}=\omega_{2}+\omega_{3}+\omega_{4}, \nu_{2}=\omega_{3}+\omega_{4}, \nu_{3}=\omega_{4}} .
$$

(a)

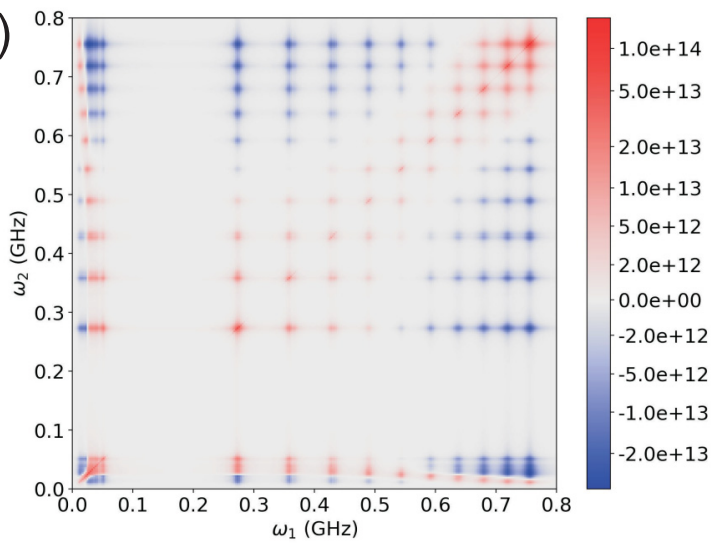

(b)

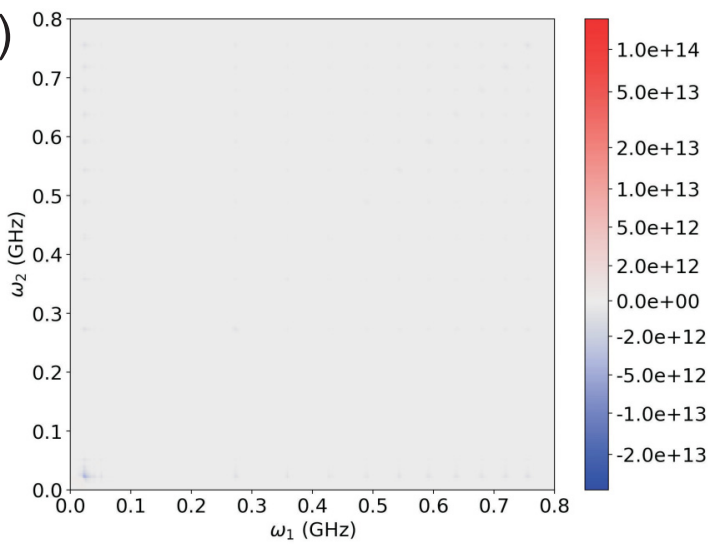

FIG. 2. (a) Replacement of Fig. $6\left(0^{\circ}\right)$ : Fourth-order correlation spectrum $S^{(4)}\left(\omega_{1},-\omega_{1}, \omega_{2},-\omega_{2}\right)$ of the spin-spin system in ZnO:In at $B=10 \mathrm{mT}$. (b) Contribution of $S^{(4,2)}+S^{(4,3)}$. 

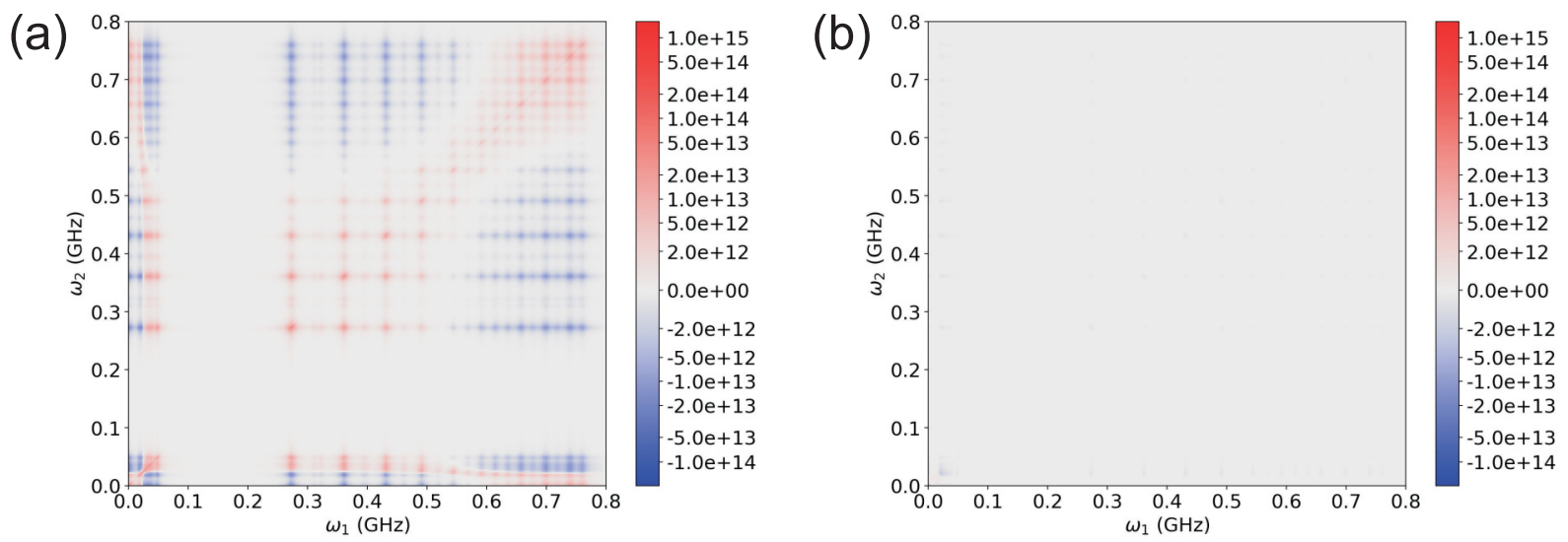

FIG. 3. (a) Replacement of Fig. $6\left(30^{\circ}\right)$ : Fourth-order correlation spectrum $S^{(4)}\left(\omega_{1},-\omega_{1}, \omega_{2},-\omega_{2}\right)$ of the spin-spin system in ZnO:In at $B=10 \mathrm{mT}$. (b) Contribution of $S^{(4,2)}+S^{(4,3)}$.

A very similar calculation for $S^{(4,2)}$ yields

$$
g_{2}\left(\tau_{1}, \tau_{2}, \tau_{3}\right)=-\beta^{8} \sum_{k \neq m, l \neq l} s_{k} s_{l} e^{\lambda_{k}\left(\tau_{3}+\tau_{2}\right)} e^{\lambda_{l}\left(\tau_{2}+\tau_{1}\right)},
$$

and, eventually,

$$
S^{(4,2)}\left(\omega_{1}, \omega_{2}, \omega_{3}, \omega_{4}\right)=\left.\beta^{8} \sum_{k \neq m, l \neq m} \sum_{\text {perm. } \omega_{j}} s_{k} s_{l} \frac{1}{\left(\lambda_{l}+i \nu_{1}\right)\left(\lambda_{k}+i \nu_{3}\right)\left(\lambda_{k}+\lambda_{l}+i \nu_{2}\right)}\right|_{\nu_{1}=\omega_{2}+\omega_{3}+\omega_{4}, \nu_{2}=\omega_{3}+\omega_{4}, \nu_{3}=\omega_{4}} .
$$

The same expression for $s_{k}$ [Eq. (6)] is used for both, $S^{(4,2)}$ and $S^{(4,3)}$ and needs to be evaluated only once for each $k$.

Application of the correct expression Eq. (109) for $S^{(4)}$ to the spin-spin system in ZnO:In leads to only small changes as compared to the incomplete previous expression. The largest change is found for $B=0 \mathrm{mT}$ where a correction at $\left(\omega_{1}, \omega_{2}\right)=$ $(0.5,0.5) \mathrm{GHz}$ occurs [Fig. 1(b)]. Very small changes occur for $B=10 \mathrm{mT}$ at out-of-plane angles of $0^{\circ}$ and $30^{\circ}$, see Figs. 2 and 3. The correction is located in the spectrum around $(0.025,0.025) \mathrm{GHz}$ and assumes both positive and negative values. The conclusions of our paper are not affected as the corrections are always very small for finite magnetic fields.

$C_{4}$ and $S^{(4)}$ are within the very general stochastic master equation approach the correct expressions for a continuous quantum measurement of the operator $\mathcal{A}$ on a system $\rho_{0}$ (equilibrium with environment and measurement apparatus) for arbitrary measurement strengths $\beta$. The corresponding expressions for $C_{2}, S^{(2)}, C_{3}$, and $S^{(3)}$ can be found in the original paper. 